\title{
Prevalence and Characteristics of Persistent Symptoms After Non-Severe COVID-19: A Prospective Cohort Study
}

\section{Lucas Armange}

CHU Rennes: Centre Hospitalier Universitaire de Rennes

\section{François Bénézit}

CHU Rennes: Centre Hospitalier Universitaire de Rennes

\section{Léa Picard}

CHU Rennes: Centre Hospitalier Universitaire de Rennes

\section{Charlotte Pronier}

CHU Rennes: Centre Hospitalier Universitaire de Rennes

\section{Stéphanie Guillot}

CHU Rennes: Centre Hospitalier Universitaire de Rennes

\section{Pierre-Axel Lentz}

CHU Rennes: Centre Hospitalier Universitaire de Rennes

\section{François Carré}

CHU Rennes: Centre Hospitalier Universitaire de Rennes

Pierre Tattevin ( $\nabla$ pierre.tattevin@chu-rennes.fr)

Pontchaillou University Hospital https://orcid.org/0000-0003-3617-5411

\section{Matthieu Revest}

CHU Rennes: Centre Hospitalier Universitaire de Rennes

\section{Research Article}

Keywords: COVID-19, persistent symptoms, prospective cohort, dyspnea, investigations, asthenia

Posted Date: February 19th, 2021

DOl: https://doi.org/10.21203/rs.3.rs-216761/v1

License: (c) (i) This work is licensed under a Creative Commons Attribution 4.0 International License. Read Full License

Version of Record: A version of this preprint was published at European Journal of Clinical Microbiology \& Infectious Diseases on April 23rd, 2021. See the published version at https://doi.org/10.1007/s10096- 
021-04261-y.

Page $2 / 10$ 


\section{Abstract}

We performed a prospective cohort study of 311 outpatients with non-severe COVID-19 (187 women, median age 39 years). Of the 214 (68.8\%) who completed the 6-week follow-up questionnaire, 115 (53.7\%) had recovered. Others mostly reported dyspnea $(n=86,40.2 \%)$, weight loss $(n=83,38.8 \%)$, sleep disorders $(n=68,31.8 \%)$, and anxiety $(n=56,26.2 \%)$. Of those who developed ageusia and anosmia, these symptoms were still present at week 6 in, respectively, 11/111 (9.9\%), and 19/114 (16.7\%). Chest CT scan and lung function tests found no explanation in the most disabled patients $(n=23)$. This study confirms the high prevalence of persistent symptoms after non-severe COVID-19.

\section{Introduction}

With > 50 million cases reported in 2020, the acute phase of coronavirus disease 2019 (COVID-19) has been well characterized, especially in patients who required hospital admission (1), but few studies focused on persistent symptoms (2-4). The characteristics and outcome of COVID-19 in patients who were not admitted have attracted less attention (5). However, physicians have been confronted with patients complaining of persistent symptoms after non-severe COVID-19 (6). We aimed to assess the prevalence of symptoms six weeks after non-severe COVID-19 in a prospective cohort of outpatients.

\section{Methods}

We conducted a monocentric observational study at the Rennes University Hospital, a tertiary-care hospital that serves as a referral center for COVID-19 in western France (population catchment area, 1.8 million inhabitants), from March, 3rd to June, 23th, 2020. We enrolled all adults outpatients diagnosed with COVID-19 in the infectious diseases ambulatory unit, who presented symptoms suggestive of COVID-19 associated with either a positive SARS-CoV-2 RT-PCR from a nasopharyngeal swab, and/or close contact with biologically-proven case(s), and who consented to participate in the study. All patients were followed through a digital application on smartphone, with daily questionnaires about symptoms, including fever, respiratory and digestive signs, pain, mood, and weight loss during the first three weeks. At any moment, they could ask questions through the application to doctors or trained medical students, or request for consultation. First day of follow-up was defined as the day of COVID-19 diagnosis.

At 6 weeks of follow-up, a link to an anonymized web-based questionnaire was sent to all patients enrolled in the prospective cohort, by e-mail. This short questionnaire collected data on respiratory symptoms, smell and taste disorders, general status, and mood (Supplementary table). Patients were offered to be contacted by one investigator (LA). For patients who were unable to resume their pre-COVID19 activities, a consultation was planned within 2 weeks, for comprehensive medical evaluation, including anamnesis and physical examination, to determine whether additional investigations and/or treatment were necessary. 
When indicated, investigations were performed within one month, and included non-contrast chest CT scan, and lung function tests (LFT). Patients who primarily complained of asthenia and motor weakness were managed in an outpatient rehabilitation center (sports medicine department). Patients with persistent smell and/or taste disorders were offered to participate in a specific rehabilitation program for anosmia/ageusia. Patients with disabling persistent respiratory symptoms were evaluated by a pulmonologist, who could initiate treatment based on LFT findings (e.g. short or long-acting bronchodilators and/or inhaled corticosteroids). Quantitative data are reported as median [interquartile range], qualitative data as number (\%). This study was approved by the Rennes university hospital institutional review board.

\section{Results}

We enrolled 311 patients, with a median age of 39 years [29-51], and a female-to-male ratio of 187/124 (1.5). Among them, 214 (68.8\%) completed the web-based questionnaire at 6 weeks (Fig. 1). Major findings at 6 -week follow-up were as follows: $115 / 214$ patients $(53.7 \%)$ had fully recovered. Others mostly reported dyspnea $(n=86,40.2 \%)$, weight loss $(n=83,38.8 \%)$, sleep disorders $(n=68,31.8 \%)$, anxiety $(n=56,26.2 \%)$, cough $(n=41,19.2 \%)$, sadness $(27,12.6 \%)$ and chest pain $(n=23,10.7 \%)$. Among the 151 patients who regularly practiced sports before their COVID-19, 84 (55.6\%) had resumed their sport activities. Among patients who suffered from smell or taste disorders during the acute phase of COVID19, these symptoms were still present at week 6 in 11/111 (9.9\%) for ageusia, and 19/114 (16.7\%) for anosmia, with a median time-to-recovery at 2 weeks (Table 1).

The 23 patients who were most disabled by respiratory symptoms underwent LFT and chest CT scan, despite their physical examination was unremarkable. They were mostly women (18/23, 78.3\%), with a median age of 44 years [34-50]. Comorbidities included asthma $(n=4)$, and smoking $(n=2)$. LFT documented decreased diffusing capacity of the lung for carbon monoxide (DLCO) in 9 patients (39.1\%), mild-to-moderate in all (mean, $64.5 \%$ normal value, minimum 56\%), including the 2 patients with active smoking. One patient had mild restrictive syndrome. Two patients presented mild desaturation during the 6-minute walk test, but never $<95 \% \mathrm{SaO}_{2}$. The median distance covered during this exercise was $90 \%$ [64-95] of theoretical values. The median Borg rating of perceived exertion scale was $5 / 10$ [3-5]. Clinical bronchial hyper-reactivity was detected in 7 patients (30.4\%), and hyperventilation syndrome suspected in three (13.0\%). Following LFT, a treatment by bronchodilators and/or inhaled corticosteroids, was introduced in 11 patients (47.8\%).

Chest CT scan did not find any pattern of pulmonary fibrosis. Ground-glass opacities were detected in 4 patients (17.4\%), significantly improved as compared with initial chest CT scan in all (Fig. 2). Of note, the 4 patients with persistent CT scan abnormalities had decreased DLCO. Fifteen patients were transferred to the sports medicine rehabilitation center, and six initiated rehabilitation program for anosmia/ageusia.

\section{Discussion}


The major findings of this prospective cohort study are the followings: i) even in patients with non-severe COVID-19, total resolution of symptoms takes time: approximately $50 \%$ of patients had not returned to their baseline health status 6 weeks after their COVID-19, and only $55 \%$ of those who practiced sports before were able to resume their sport activities; ii) the primary complaints, at 6 weeks, included dyspnea, weight loss, sleep disorders, and anxiety; iii) even among patients most affected by post-COVID symptoms, physical examination was unremarkable, while LFT and chest CT scan were normal in most cases.

Little is known about the persistence of disabling symptoms following COVID-19. A post-acute outpatient service for individuals discharged from hospital after recovery from COVID-19 in Italy found that only 18/143 patients (12.6\%) were completely free of COVID-19-related symptoms at 60 days. Fatigue, dyspnea, pain, and lack of appetite, were reported by $>50 \%$ of patients (2). Similar findings were reported from Ireland, where $52.3 \%$ of patients (67/128) reported persistent fatigue 10 weeks after COVID-19, and this was not associated with severity during the acute COVID-19 (3). A multicenter study from Egypt found higher prevalence of fatigue in patients who recovered from COVID-19 (209/287, $72.8 \%$ ) (4). To our knowledge, our study is the first to provide systematic investigations in patients most disabled by respiratory symptoms: All of them (23/23) had normal physical examination, no new findings on chest CT-scan, and no or limited abnormalities on LFT.

The optimal management of patients with persistent symptoms after the acute phase of COVID-19 remains unknown. A Post-COVID-19 Functional Status (PCFS) scale has been proposed to evaluate the entire range of functional limitations, including in lifestyle, sports and social activities (7). Others advocated for an interdisciplinary approach, through a post-COVID outpatient unit, to implement comprehensive and individualized care for these patients $(6,8,9)$.

Our study has limitations. First, our web-based questionnaire was deliberately short, to ensure a high proportion of answers: hence, it was not powered to detect all post-COVID symptoms. Second, we only collected data at 6 weeks, so that we have no information beyond this time point. Additional studies are requested to estimate symptoms duration, as this is one of the main concerns for patients. Third, investigations were restricted to patients most disabled, and were limited to non-contrast chest CT scan and LFT. However, in our experience, other investigations, including contrast-enhanced CT scan, echocardiography, and tests for inflammatory or auto-immune disorders, provide no additional information about the pathophysiology of persistent symptoms.

In conclusion, this prospective cohort study confirms the high prevalence of persistent symptoms even after non-severe COVID-19. Long-term follow-up with multidisciplinary management is requested to assist patients throughout this difficult recovery time.

\section{Declarations}

FUNDING. No funding was received for this study 
COMPETING INTERESTS. All authors: none

ETHICAL APPROVAL. The study was approved by the Ethic Committee of Rennes University Hospital.

Consent to participate. Waived.

Consent to publish. Waived.

Availability of data and materials. On request.

Code availability. Not applicable.

Authors contribution. LA, FB, MR and PT participated in study design, collected data, and wrote the first draft of the manuscript. All authors were involved in patients care, revised and approved the final version of the manuscript.

\section{References}

1. Zhou F, Yu T, Du R, Fan G, Liu Y, Liu Z, et al. Clinical course and risk factors for mortality of adult inpatients with COVID-19 in Wuhan, China: a retrospective cohort study. The Lancet. 2020 Mar;395(10229):1054-62.

2. Carfi A, Bernabei R, Landi F, for the Gemelli Against COVID-19 Post-Acute Care Study Group. Persistent Symptoms in Patients After Acute COVID-19. JAMA [Internet]. 2020 Jul 9 [cited 2020 Jul 13]; Available from: https://jamanetwork.com/journals/jama/fullarticle/2768351

3. Townsend L, Dyer AH, Jones K, Dunne J, Mooney A, Gaffney F, et al. Persistent fatigue following SARS-CoV-2 infection is common and independent of severity of initial infection. PLoS One. 2020;15(11):e0240784.

4. Kamal M, Abo Omirah M, Hussein A, Saeed H. Assessment and characterisation of post-COVID-19 manifestations. Int J Clin Pract. 2020 Sep 29;e13746.

5. Lutchmansingh DD, Knauert MP, Antin-Ozerkis DE, Chupp G, Cohn L, Dela Cruz CS, et al. A clinic blueprint for post-COVID-19 RECOVERY: Learning from the past, looking to the future. Chest. 2020 Nov 4;

6. Landi F, Gremese E, Bernabei R, Fantoni M, Gasbarrini A, Settanni CR, et al. Post-COVID-19 global health strategies: the need for an interdisciplinary approach. Aging Clin Exp Res. 2020 Aug 1;32(8):1613-20.

7. Klok FA, Boon GJAM, Barco S, Endres M, Geelhoed JJM, Knauss S, et al. The Post-COVID-19 Functional Status scale: a tool to measure functional status over time after COVID-19. Eur Respir J. 2020;56(1).

8. Barker-Davies RM, O'Sullivan O, Senaratne KPP, Baker P, Cranley M, Dharm-Datta S, et al. The Stanford Hall consensus statement for post-COVID-19 rehabilitation. Br J Sports Med. 2020 Aug;54(16):949-59. 
9. Davido B, Seang S, Tubiana R, de Truchis P. Post-COVID-19 chronic symptoms: a postinfectious entity? Clin Microbiol Infect. 2020 Jul 23;

\section{Tables}

Table 1. Non-severe COVID-19 patients baseline characteristics and 6-week follow-up 


\begin{tabular}{|ll|}
\hline Baseline (n=311) & \\
\hline Age, years & $39[29-51]$ \\
\hline Female sex & $187(60.1 \%)$ \\
\hline At least one comorbidity & $61(19.6 \%)$ \\
\hline Active smoking & $25(8.0 \%)$ \\
\hline Chronic respiratory disease & $25(8.0 \%)$ \\
\hline Chronic heart disease & $14(4.5 \%)$ \\
\hline Six-week health status (n=214) & \\
\hline Returned to baseline health status, no residual symptom & $115(53.7 \%)$ \\
\hline Dyspnea & $86(40.2 \%)$ \\
\hline - Only on exertion & $76(35.6 \%)$ \\
\hline - Permanent & $10(4.7 \%)$ \\
\hline Cough & $41(19.2 \%)$ \\
\hline Chest pain & $23(10.7 \%)$ \\
\hline Weight loss & $83(38.8 \%)$ \\
\hline Sleep disorders & $68(31.8 \%)$ \\
\hline Anxiety & $56(26.2 \%)$ \\
\hline Sadness & $27(12.6 \%)$ \\
\hline Respiratory functional explorations (n=23) & 0 \\
\hline Decreased DLCO & $7 / 23(39 \%)$ \\
\hline Clinical bronchial hyper-reactivity & $1 / 23(4 \%)$ \\
\hline Restrictive syndrome & $0 / 23(0 \%)$ \\
\hline Obstructive syndrome & \\
\hline Chest CT scan findings (n=23) & \\
\hline Ground glass lesions * & \\
\hline Pulmonary fibrosis & \\
\hline
\end{tabular}

Quantitative data are reported as median [interquartile range], qualitative data as number (\%) DLCO, diffusing capacity of the lung for carbon monoxide 
* in all cases (4/4), lesions were significantly improved as compared to baseline CT scan

\section{Figures}

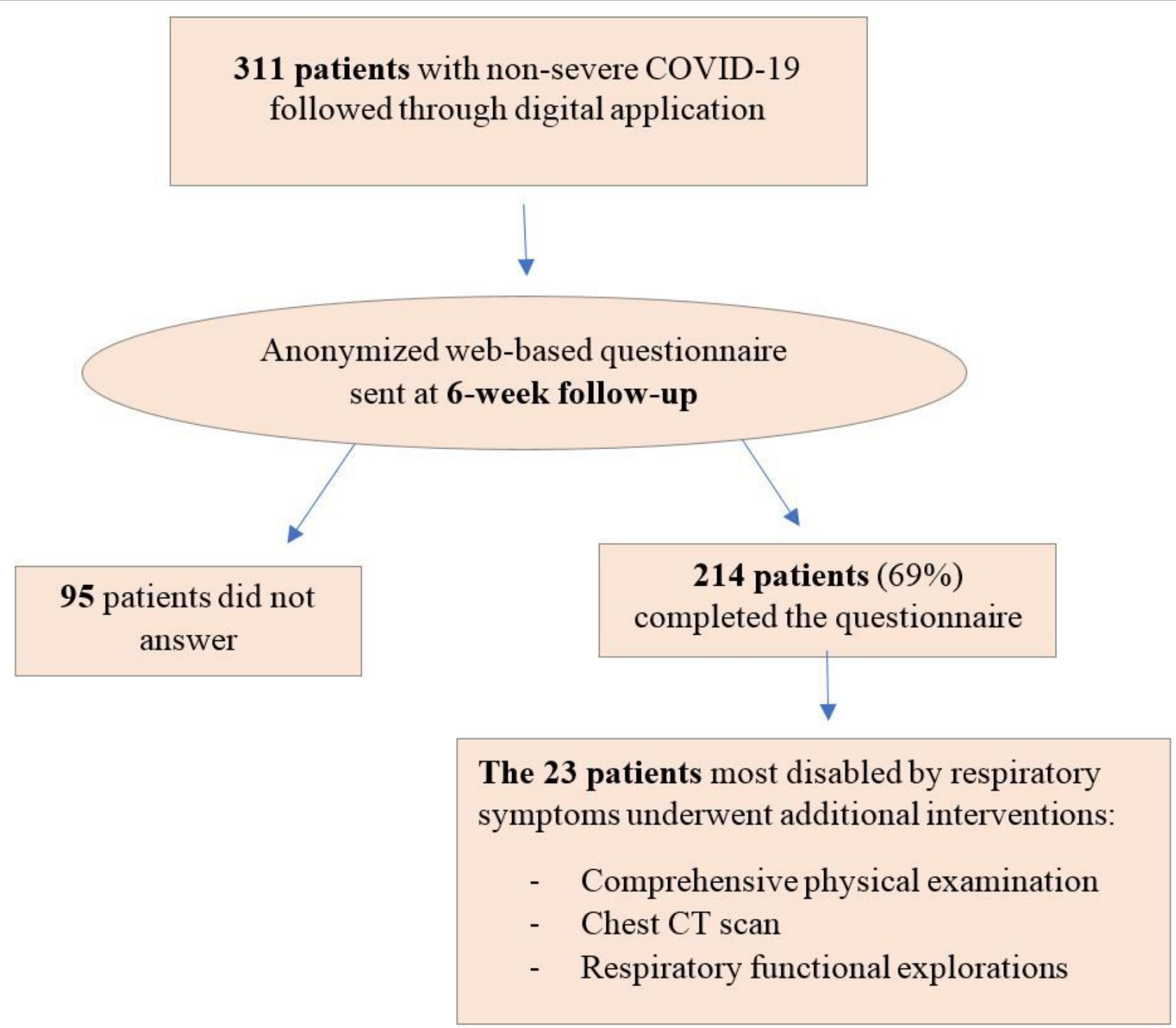

Figure 1

Study flowchart. 

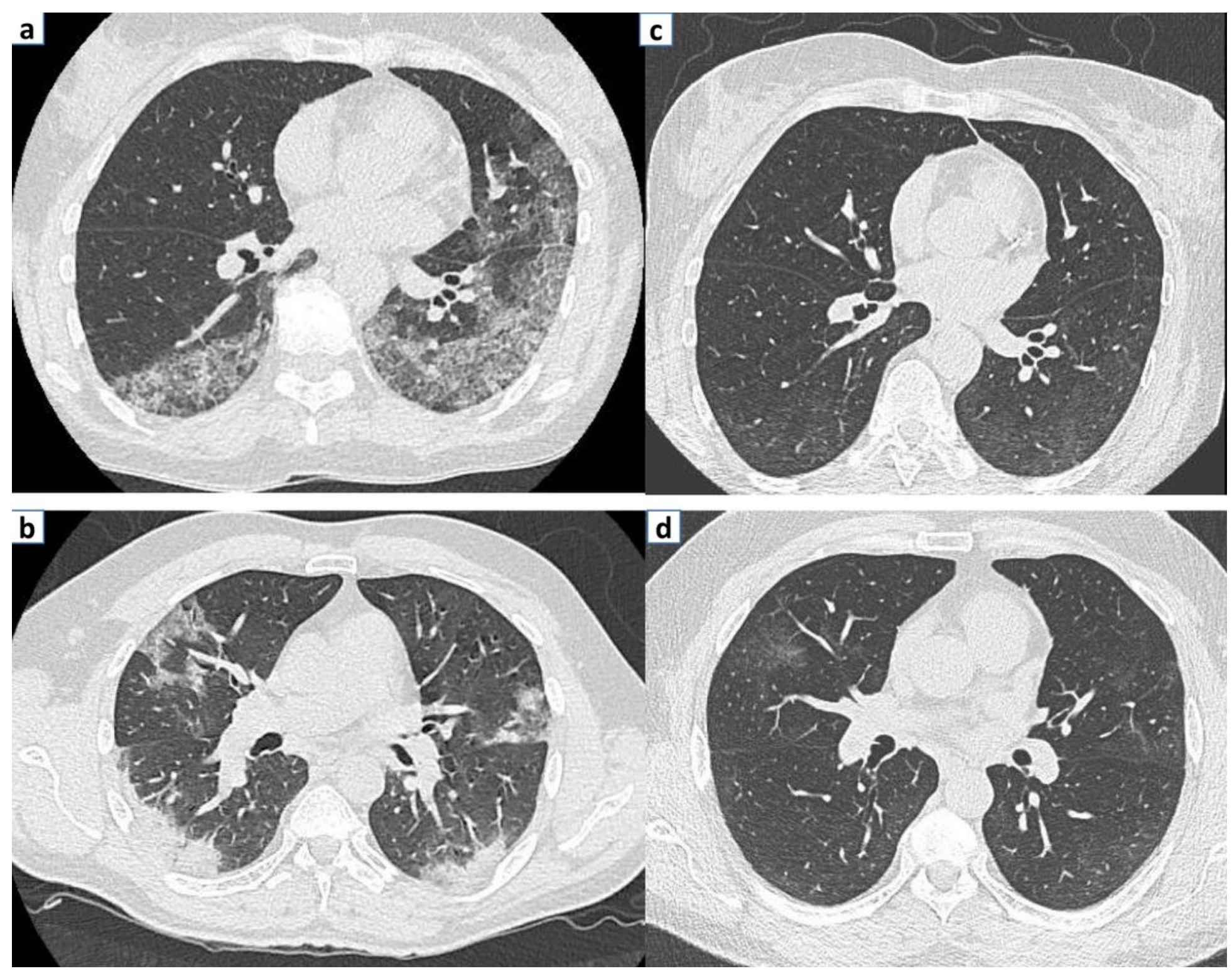

Figure 2

Non-contrast chest CT scan 2a, 2b. Consolidations and ground-glass opacities with crazy paving at baseline in 2 patients. $2 \mathrm{c}, 2 \mathrm{~d}$. Same patients, control at 10 weeks with nearly complete resolution 